\title{
HODGE INDEX THEOREM FOR ARITHMETIC CYCLES OF CODIMENSION ONE
}

\author{
Atsushi MoriWAKI
}

\section{Introduction}

Let $f: X \rightarrow \operatorname{Spec}(\mathbb{Z})$ be a $(d+1)$-dimensional regular arithmetic variety over $\operatorname{Spec}(\mathbb{Z})$, i.e. $X$ is regular, $X$ is projective and flat over $\operatorname{Spec}(\mathbb{Z})$ and $d=\operatorname{dim} f$. Let $H$ be an $f$-ample line bundle on $X$ and $k$ a Hermitian metric of $H$. Here we consider a homomorphism

$$
L: \widehat{\mathrm{CH}}^{p}(X)_{\mathbb{R}} \rightarrow \widehat{\mathrm{CH}}^{p+1}(X)_{\mathbb{R}}
$$

defined by $L(x)=x \cdot \widehat{c}_{1}(H, k)$. In [GS], H. Gillet and C. Soulé conjectured that

Arithmetic Analogues of Grothendieck's Standard Conjectures. For a suitable choice of $k$, if $2 p \leq d+1$, then

(a) The homomorphism $L^{d+1-2 p}: \widehat{\mathrm{CH}}^{p}(X)_{\mathbb{R}} \rightarrow \widehat{\mathrm{CH}}^{d+1-p}(X)_{\mathbb{R}}$ is bijective, and

(b) If $x \in \widehat{\mathrm{CH}}^{p}(X)_{\mathbb{R}}, \quad x \neq 0$ and $L^{d+2-2 p}(x)=0$, then $(-1)^{p} \widehat{\operatorname{deg}}\left(x \cdot L^{d+1-2 p}(x)\right)>0$.

For example, K. Künnemann [Ku] proved that if $X$ is a projective space, then the conjecture is true. Here we fix a notation. We say a Hermitian line bundle $(H, k)$ on $X$ is arithmetically ample if (1) $H$ is $f$-ample, (2) the Chern form $c_{1}\left(H_{\infty}, k_{\infty}\right)$ is positive definite on the infinite fiber $X_{\infty}$, and (3) there is a positive integer $m_{0}$ such that, for any integer $m \geq m_{0}$, $H^{0}\left(X, H^{m}\right)$ is generated by the set $\left\{s \in H^{0}\left(X, H^{m}\right) \mid\left\|s_{\infty}\right\|_{\text {sup }}<1\right\}$. Note that by virtue of $[\mathrm{Zh}]$, the third condition can be replaced by a numerical condition : (3)' for every irreducible horizontal subvariety $Y$ (i.e. $Y$ is flat over $\operatorname{Spec}(\mathbb{Z}))$, the height $\widehat{c}_{1}\left(\left.(H, k)\right|_{Y}\right)^{\operatorname{dim} Y}$ of $Y$ is positive. In this note, we would like to prove the following partial answer of the above conjecture for general regular arithmetic varieties.

Received December 6, 1995. 
Theorem A. Assume that $d \geq 1$ and $(H, k)$ is arithmetically ample. Then we have the following:

(1) $L^{d-1}: \widehat{\mathrm{CH}}^{1}(X)_{\mathbb{R}} \rightarrow \widehat{\mathrm{CH}}^{d}(X)_{\mathbb{R}}$ is injective.

(2) If $x \in \widehat{\mathrm{CH}}^{1}(X)_{\mathbb{R}}, x \neq 0$ and $L^{d}(x)=0$, then $\widehat{\operatorname{deg}}\left(x L^{d-1}(x)\right)<0$.

Theorem A is a consequence of the following higher dimensional generalization of Faltings-Hriljac's Hodge index theorem on arithmetic surfaces (cf. $[\mathrm{Fa}]$ and $[\mathrm{Hr}])$.

Theorem B. Assume that $d \geq 1$ and $(H, k)$ is arithmetically ample. Let $X \stackrel{f^{\prime}}{\longrightarrow} \operatorname{Spec}\left(O_{K}\right) \rightarrow \operatorname{Spec}(\mathbb{Z})$ be the Stein factorization of $f: X \rightarrow$ $\operatorname{Spec}(\mathbb{Z})$ and $X_{K}$ the generic fiber of $f^{\prime}$, where $O_{K}$ is the ring of integers of an algebraic number field $K$. Let $z: \widehat{\mathrm{CH}}^{1}(X) \rightarrow \mathrm{CH}^{1}(X)$ be the canonical homomorphism defined by $z(D, g)=D$. If $x \in \widehat{\mathrm{CH}}^{1}(X)$ and $\left(\left.z(x)\right|_{X_{K}} \cdot\left(\left.H\right|_{X_{K}}\right)^{d-1}\right)=0$, then

$$
\widehat{\operatorname{deg}}\left(x^{2} \cdot \widehat{c}_{1}(H, k)^{d-1}\right) \leq 0 .
$$

Moreover, equality holds if and only if there are a positive integer $n$ and $y \in \widehat{\mathrm{CH}}^{1}\left(\operatorname{Spec}\left(O_{K}\right)\right)$ such that $n x=f^{\prime *}(y)$.

\section{Proof of Theorem B}

In this section, we would like to give the proof of Theorem B. An advantage to use arithmetical ampleness of the Hermitian line bundle $(H, k)$ is the following arithmetic Bertini's theorem.

Arithmetic Bertini's Theorem. (cf. [Mo2, Theorem 4.2 and Theorem 5.2]) Let $f: X \rightarrow \operatorname{Spec}(\mathbb{Z})$ be an arithmetic variety, and $(H, k)$ an arithmetically ample Hermitian line bundle on $X$. If $x_{1}, \ldots, x_{s}$ are points (not necessarily closed) on $X$, then, for a sufficiently large integer $m$, there is a section $\phi$ of $H^{0}\left(X, H^{m}\right)$ such that

(1) $\operatorname{div}(\phi)$ is smooth over $\mathbb{Q}$,

(2) $\phi\left(x_{i}\right) \neq 0$ for all $1 \leq i \leq s$, and

(3) $\left\|\phi_{\infty}\right\|_{\text {sup }}<1$.

By the above theorem, we can proceed to induction on $d=\operatorname{dim} f$. However, regularity of $X$ doesn't preserve by induction step in general. Here we consider the following weaker version on general arithmetic varieties. 
Theorem 1.1. Let $K$ be an algebraic number field and $O_{K}$ the ring of integers of $K$. Let $f: X \rightarrow \operatorname{Spec}\left(O_{K}\right)$ be an arithmetic variety such that $d=\operatorname{dim} f \geq 1$ and $X_{K}$ is smooth and geometrically irreducible. Let $(H, k)$ be an arithmetically ample Hermitian line bundle on $X$. Let $D$ be a Cartier divisor on $X$ and $g_{\sigma}$ a Green current of $D_{\sigma}$ on each $\sigma \in K(\mathbb{C})$. If $\left(D_{K}\right.$. $\left.H_{K}^{d-1}\right)=0$, then

$$
\widehat{\operatorname{deg}}\left(\left(D, \sum g_{\sigma}\right)^{2} \cdot \widehat{c}_{1}(H, k)^{d-1}\right) \leq 0
$$

Moreover, if equality holds, then there are a positive integer n, a Cartier divisor $Z$ on $X$ and constants $\left\{g_{\sigma}^{\prime}\right\}_{\sigma \in K(\mathbb{C})}$ such that the support of $Z$ is vertical and the class of $\left(Z, \sum g_{\sigma}^{\prime}\right)$ is equal to the class of $n\left(D, \sum g_{\sigma}\right)$ in $\widehat{\mathrm{CH}}^{1}(X)$. In particular, if equality holds, then $\mathcal{O}_{X_{K}}\left(D_{K}\right)$ is a torsion of $\operatorname{Pic}\left(X_{K}\right)$.

Proof. First of all, we prepare two lemmas.

Lemma 1.1.1. Let $X$ be a d-dimensional compact Kähler manifold with a Kähler form $\Phi$ and $\varphi$ a real valued smooth function on $X$. Then,

$$
\int_{X} \varphi d d^{c}(\varphi) \Phi^{d-1} \leq 0
$$

Moreover, equality holds if and only if $\varphi$ is a constant.

Proof. Since $d d^{c}=\frac{\sqrt{-1}}{2 \pi} \partial \bar{\partial}$ and $d(\varphi \bar{\partial}(\varphi))=\partial(\varphi) \bar{\partial}(\varphi)+\varphi \partial \bar{\partial}(\varphi)$, by Stokes' theorem, we have

$$
\int_{X} \varphi d d^{c}(\varphi) \Phi^{d-1}=-\frac{\sqrt{-1}}{2 \pi} \int_{X} \partial(\varphi) \bar{\partial}(\varphi) \Phi^{d-1} .
$$

Here let $\theta^{1}, \ldots, \theta^{d}$ be a local unitary frame of $\Omega_{X}^{1}$ with $\Phi=\sqrt{-1} \sum_{i} \theta^{i} \wedge \bar{\theta}^{i}$. We set $\partial(\varphi)=\sum_{i} a_{i} \theta^{i}$. Then, $\bar{\partial}(\varphi)=\overline{\partial(\varphi)}=\sum_{i} \bar{a}_{i} \bar{\theta}^{i}$. Therefore,

$$
-\frac{\sqrt{-1}}{2 \pi} \partial(\varphi) \bar{\partial}(\varphi) \Phi^{d-1}=\frac{-1}{2 \pi} \sum_{i=1}^{d}\left|a_{i}\right|^{2} \Phi^{d} .
$$

Thus, we have

$$
\int_{X} \varphi d d^{c}(\varphi) \Phi^{d-1} \leq 0
$$

Moreover, equality hold if and only if $\partial(\varphi)=0$. Here, since $\varphi$ is real valued, $\partial(\varphi)=0$ implies that $\varphi$ is a constant. 
Lemma 1.1.2. Let $X$ be a d-dimensional Kähler manifold with a Kähler form $\Phi$ and $\omega$ a smooth $(1,1)$-form on $X$ such that $\bar{\omega}=-\omega$ and $\omega \wedge \Phi^{d-1}=$ 0 . Then, there is a real valued smooth function $u$ on $X$ with the following properties.

(1) $\omega^{2} \wedge \Phi^{d-2}=u \Phi^{d}$.

(2) $u(x) \leq 0$ for all $x \in X$.

(3) $u(x)=0$ for all $x \in X$ if and only if $\omega=0$.

Proof. Let $\theta^{1}, \ldots, \theta^{d}$ be a local unitary frame of $\Omega_{X}^{1}$ with $\Phi=\sqrt{-1} \sum_{i} \theta^{i} \wedge$ $\bar{\theta}^{i}$. We set $\omega=\sum_{i, j} a_{i j} \theta^{i} \wedge \bar{\theta}^{j}$. Then, $\bar{\omega}=-\omega$ implies that $a_{j i}=-\bar{a}_{i j}$. Moreover, since

$$
\omega \wedge \Phi^{d-1}=-\sqrt{-1}\left(a_{11}+\cdots+a_{d d}\right) \Phi^{d}
$$

we have $a_{11}+\cdots+a_{d d}=0$. On the other hand, by an easy calculation,

$$
d(d-1) \omega^{2} \wedge \Phi^{d-2}=\left(\sum_{i, j} a_{i j} a_{j i}-a_{i i} a_{j j}\right) \Phi^{d} .
$$

Therefore, we get

$$
\omega^{2} \wedge \Phi^{d-2}=\frac{-1}{d(d-1)}\left(\sum_{i, j}\left|a_{i j}\right|^{2}\right) \Phi^{d} .
$$

Hence, if we set $u=\frac{-1}{d(d-1)} \sum_{i, j}\left|a_{i j}\right|^{2}$, the lemma is obtained because $\sum_{i, j}\left|a_{i j}\right|^{2}$ is independent of the choice of $\theta^{1}, \ldots, \theta^{d}$.

Let us start of the proof of Theorem 1.1. We will prove it by induction on $d$. First, we consider the case $d=1$. In this case, taking a desingularization of $X$, we may assume that $X$ is regular. Thus, our theorem can be derived from Faltings-Hriljac's Hodge index theorem (cf. [Fa] and $[\mathrm{Hr}]$ ).

Next, we assume $d \geq 2$. We set $x=\left(D, \sum g_{\sigma}\right)$ and $L=\mathcal{O}_{X}(D)$. Let $h_{\sigma}$ be an Einstein-Hermitian metric of $L_{\sigma}$ with respect to $c_{1}\left(H_{\sigma}, k_{\sigma}\right)$. Let $s$ be a rational section of $L$ with $\operatorname{div}(s)=D$. Here we consider an arithmetic cycle

$$
y=\left(D, \sum_{\sigma \in K(\mathbb{C})}-\log \left(h_{\sigma}\left(s_{\sigma}, s_{\sigma}\right)\right)\right) .
$$


Since $g_{\sigma}$ and $-\log \left(h_{\sigma}\left(s_{\sigma}, s_{\sigma}\right)\right)$ are Green currents of the same $D_{\sigma}$, there is a real valued smooth function $\phi_{\sigma}$ on each $X_{\sigma}$ such that $x=y+a\left(\sum_{\sigma \in K(\mathbb{C})} \phi_{\sigma}\right)$ in $\widehat{\mathrm{CH}}^{1}(X)$. Then, it is easy to see that

$$
\begin{aligned}
\widehat{\operatorname{deg}}\left(x^{2} \cdot \widehat{c}_{1}(H, k)^{d-1}\right)= & \widehat{\operatorname{deg}}\left(y^{2} \cdot \widehat{c}_{1}(H, k)^{d-1}\right)+ \\
& \frac{1}{2} \sum_{\sigma \in K(\mathbb{C})} \int_{X_{\sigma}} \phi_{\sigma} d d^{c}\left(\phi_{\sigma}\right) c_{1}\left(H_{\sigma}, k_{\sigma}\right)^{d-1}
\end{aligned}
$$

because $c_{1}\left(L_{\sigma}, h_{\sigma}\right) c_{1}\left(H_{\sigma}, k_{\sigma}\right)^{d-1}=0$. Therefore, by Lemma 1.1.1,

$$
\widehat{\operatorname{deg}}\left(x^{2} \cdot \widehat{c}_{1}(H, k)^{d-1}\right) \leq \widehat{\operatorname{deg}}\left(y^{2} \cdot \widehat{c}_{1}(H, k)^{d-1}\right)
$$

and equality holds if and only if $\phi_{\sigma}$ is a constant for each $\sigma \in K(\mathbb{C})$. On the other hand, by virtue of arithmetic Bertini's theorem, for a sufficiently large $m$, there is a section $t \in H^{0}\left(X, H^{m}\right)$ with the following properties:

i) $\operatorname{div}(t)_{K}$ is smooth and geometrically irreducible.

ii) If $\operatorname{div}(t)=Y+a_{1} F_{1}+\cdots+a_{s} F_{s}$ is the irreducible decomposition such that $Y$ is horizontal and $F_{i}$ 's are vertical, then $F_{i}$ 's are smooth fibers.

iii) $D$ and $\operatorname{div}(t)$ has no common irreducible component.

iv) $\sup _{x \in X_{\sigma}}\left(\left\|t_{\sigma}\right\|_{k_{\sigma}^{m}}(x)\right)<1$ for all $\sigma \in K(\mathbb{C})$.

(Note that $H^{1}\left(X_{K}, H_{K}^{-m}\right)=0$ guarantees geometrical irreducibility of $\left.\operatorname{div}(t)_{K} \cdot\right)$ Since $\left(\left.\left.D\right|_{F_{i}} ^{2} \cdot H\right|_{F_{i}} ^{d-2}\right) \leq 0$ by the geometric Hodge index theorem, we obtain

$$
\begin{aligned}
\widehat{\operatorname{deg}}\left(y^{2} \cdot \widehat{c}_{1}\left(H^{m}, k^{m}\right)^{d-1}\right)= & \widehat{\operatorname{deg}}\left(\left.y\right|_{Y} ^{2} \cdot \widehat{c}_{1}\left(\left.\left(H^{m}, k^{m}\right)\right|_{Y}\right)^{d-2}\right)+ \\
& \sum a_{i} m\left(\left.\left.D\right|_{F_{i}} ^{2} \cdot H\right|_{F_{i}} ^{d-2}\right)- \\
& \sum_{\sigma \in K(\mathbb{C})} \int_{X_{\sigma}} \log \left(\left\|t_{\sigma}\right\|_{k_{\sigma}^{m}}\right) c_{1}\left(L_{\sigma}, h_{\sigma}\right)^{2} c_{1}\left(H_{\sigma}^{m}, k_{\sigma}^{m}\right)^{d-2} \\
\leq & \widehat{\operatorname{deg}}\left(\left.y\right|_{Y} ^{2} \cdot \widehat{c}_{1}\left(\left.\left(H^{m}, k^{m}\right)\right|_{Y}\right)^{d-2}\right)- \\
& \sum_{\sigma \in K(\mathbb{C})} \int_{X_{\sigma}} \log \left(\left\|t_{\sigma}\right\|_{k_{\sigma}^{m}}\right) c_{1}\left(L_{\sigma}, h_{\sigma}\right)^{2} c_{1}\left(H_{\sigma}^{m}, k_{\sigma}^{m}\right)^{d-2} .
\end{aligned}
$$

Since $\left(L_{\sigma}, h_{\sigma}\right)$ is Einstein-Hermitian, by Lemma 1.1.2, there is a real-valued smooth function $u_{\sigma}$ on $X_{\sigma}$ with the following properties:

(1) $c_{1}\left(L_{\sigma}, h_{\sigma}\right)^{2} c_{1}\left(H_{\sigma}, k_{\sigma}\right)^{d-2}=u_{\sigma} c_{1}\left(H_{\sigma}, k_{\sigma}\right)^{d}$.

(2) $u_{\sigma}(x) \leq 0$ for all $x \in X_{\sigma}$.

(3) $u_{\sigma}(x)=0$ for all $x \in X_{\sigma}$ if and only if $\left(L_{\sigma}, h_{\sigma}\right)$ is flat. 
Therefore, we have

$$
\widehat{\operatorname{deg}}\left(y^{2} \cdot \widehat{c}_{1}(H, k)^{d-1}\right) \leq \widehat{\operatorname{deg}}\left(\left(\left.y\right|_{Y}\right)^{2} \cdot \widehat{c}_{1}\left(\left.(H, k)\right|_{Y}\right)^{d-2}\right) .
$$

Hence, by hypothesis of induction, we get our inequality.

Finally, we consider the equality condition. We assume $\widehat{\operatorname{deg}}\left(x^{2} \cdot \widehat{c}_{1}(H, k)^{d-1}\right)=0$. Then, if we trace back the above proof carefully, we can see

(a) $\phi_{\sigma}$ is a constant for each $\sigma \in K(\mathbb{C})$.

(b) $\left(L_{\sigma}, h_{\sigma}\right)$ is flat for each $\sigma \in K(\mathbb{C})$.

(c) $\left.L_{K}\right|_{Y_{K}}$ is a torsion of $\operatorname{Pic}\left(Y_{K}\right)$.

By (b), $L_{\mathbb{C}}$ is given by a representation $\rho: \pi_{1}\left(X_{\mathbb{C}}\right) \rightarrow \mathbb{C}^{*}$ of the fundamental group of $X_{\mathbb{C}}$. (c) implies that the image of $\pi_{1}\left(Y_{\mathbb{C}}\right) \rightarrow \pi_{1}\left(X_{\mathbb{C}}\right) \rightarrow \mathbb{C}^{*}$ is finite. On the other hand, by Lefschetz theorem (cf. Theorem 7.4 in [Mi]), $\pi_{1}\left(Y_{\mathbb{C}}\right) \rightarrow \pi_{1}\left(X_{\mathbb{C}}\right)$ is surjective. Thus, the image of $\rho: \pi_{1}\left(X_{\mathbb{C}}\right) \rightarrow \mathbb{C}^{*}$ is also finite. Therefore, there is a positive integer $n$ with $L_{\mathbb{C}}^{n} \simeq \mathcal{O}_{X_{\mathbb{C}}}$. Thus,

$$
\operatorname{dim}_{K} H^{0}\left(X_{K}, L_{K}^{n}\right)=\operatorname{dim}_{\mathbb{C}} H^{0}\left(X_{K}, L_{K}^{n}\right) \otimes \mathbb{C}=\operatorname{dim}_{\mathbb{C}} H^{0}\left(X_{\mathbb{C}}, L_{\mathbb{C}}^{n}\right)=1 .
$$

Hence, since $\left(L_{K} \cdot H_{K}^{d-1}\right)=0$, we have $L_{K}^{n} \simeq \mathcal{O}_{X_{K}}$. Thus, there is a rational section $s^{\prime}$ of $L^{n}$ with $s_{K}^{\prime}=1$. We set $Z=\operatorname{div}\left(s^{\prime}\right)$ and $g_{\sigma}^{\prime}=$ $-\log \left(h_{\sigma}^{n}\left(s^{\prime}, s^{\prime}\right)\right)+n \phi_{\sigma}$. Then, the support of $Z$ is vertical. Moreover, since $h_{\sigma}^{n}$ is a flat metric of $\mathcal{O}_{X_{\sigma}}, h_{\sigma}^{n}\left(s^{\prime}, s^{\prime}\right)$ must be a constant. Therefore, $\left(Z, \sum g_{\sigma}^{\prime}\right)$ is our desired cycle.

Proof of Theorem B. Since $f_{*}^{\prime} \mathcal{O}_{X}=O_{K}, X_{K}$ is geometrically irreducible. So the inequality is an immediate consequence of Theorem 1.1.

We need to consider the precise equality condition. Clearly, if there are a positive integer $n$ and $y \in \widehat{\mathrm{CH}}^{1}\left(\operatorname{Spec}\left(O_{K}\right)\right)$ such that $n x=f^{\prime *}(y)$, then $\widehat{\operatorname{deg}}\left(x^{2} \cdot \widehat{c}_{1}(H, k)^{d-1}\right)=0$. Conversely we assume $\widehat{\operatorname{deg}}\left(x^{2} \cdot \widehat{c}_{1}(H, k)^{d-1}\right)=0$. Then, by Theorem 1.1, there are a positive integer $n_{1}$ and an arithmetic cycle $\left(Z, \sum_{\sigma \in K(\mathbb{C})} g_{\sigma}\right)$ such that $Z$ is vertical with respect to $f^{\prime}, g_{\sigma}$ 's are constant and $n_{1} x$ is equal to the class of $\left(Z, \sum_{\sigma \in K(\mathbb{C})} g_{\sigma}\right)$ in $\widehat{\mathrm{CH}}^{1}(X)$. Then,

$$
\widehat{\operatorname{deg}}\left(\left(n_{1} x\right)^{2} \cdot \widehat{c}_{1}(H, k)^{d-1}\right)=\left(Z^{2} \cdot H^{d-1}\right)=0 .
$$

Here, we need the following lemma.

Lemma 1.3. Let $X$ be a regular scheme, $R$ a discrete valuation ring, $f: X \rightarrow \operatorname{Spec}(R)$ a projective morphism with $f_{*} \mathcal{O}_{X}=R$, and $H$ an $f$ ample line bundle on $X$. Let $X_{o}$ be the central fiber of $f$ and $\left(X_{o}\right)_{\text {red }}=$ 
$X_{1}+\cdots+X_{n}$ the irreducible decomposition of $\left(X_{o}\right)_{\mathrm{red}}$. We consider a vector space $V=\bigoplus_{i=1}^{n} \mathbb{Q} X_{i}$ generated by $X_{i}$ 's and the natural pairing $():, V \times V \rightarrow \mathbb{Q}$ defined by

$$
\left(D_{1}, D_{2}\right)=\left(D_{1} \cdot D_{2} \cdot H^{d-1}\right),
$$

where $d=\operatorname{dim} f$ and $\cdot$ is the intersection product. Then, we have $(D, D) \leq$ 0 for all $D \in V$ and equality holds if and only if $D \in \mathbb{Q} X_{o}$.

Proof. For example, see (i)' of Lemma (2.10) in Chap. I of [BPV].

By the above lemma, there is a positive integer $n_{2}$ and a cycle $T$ on $\operatorname{Spec}\left(\mathcal{O}_{K}\right)$ such that $n_{2} Z=f^{\prime *}(T)$. Therefore, if we set $y=\left(T, \sum_{\sigma \in K(\mathbb{C})} n_{2} g_{\sigma}\right)$, then $n_{1} n_{2} x=f^{\prime *}(y)$.

\section{Proof of Theorem A}

Let us begin the proof of Theorem A. This is an easy corollary of Theorem B.

(1) Let us see that (2) implies (1). Assume that $L^{d-1}(x)=0$. Then, $L^{d}(x)=0$. Thus if $x \neq 0$, then $\widehat{\operatorname{deg}}\left(x L^{d-1}(x)\right)<0$ by $(2)$. This is a contradiction. Therefore, $x=0$.

(2) Let $X \stackrel{f^{\prime}}{\longrightarrow} \operatorname{Spec}\left(O_{K}\right) \rightarrow \operatorname{Spec}(\mathbb{Z})$ be the Stein factorization of $f$ : $X \rightarrow \operatorname{Spec}(\mathbb{Z})$. In the following arguments, the subscript $K$ means the restriction to the generic fiber of $f^{\prime}$.

Since $x$ can be approximated by points $y \in \widehat{\mathrm{CH}}^{1}(X)_{\mathbb{Q}}$ with $L^{d}(y)=0$, we may assume that $x \in \widehat{\mathrm{CH}}^{1}(X)_{\mathbb{Q}}$. Let $t$ be a rational number with $\left(z(x)_{K}+t H_{K} \cdot H_{K}^{d-1}\right)=0$. Replacing $x$ by $m x$, we may assume that $x \in \widehat{\mathrm{CH}}^{1}(x)$ and $t \in \mathbb{Z}$. We set $y=x+t \widehat{c}_{1}(H, k)$. Then, $\left(z(y)_{K} \cdot H_{K}^{d-1}\right)=0$. Thus, by Theorem B, we have $\widehat{\operatorname{deg}}\left(y^{2} \cdot \widehat{c}_{1}(H, k)^{d-1}\right) \leq 0$. Therefore, since $L^{d}(x)=0$, we get

$$
\widehat{\operatorname{deg}}\left(x^{2} \cdot \widehat{c}_{1}(H, k)^{d-1}\right)+(t)^{2} \widehat{\operatorname{deg}}\left(\widehat{c}_{1}(H, k)^{d+1}\right) \leq 0 .
$$

Hence, $\widehat{\operatorname{deg}}\left(x^{2} \cdot \widehat{c}_{1}(H, k)^{d-1}\right) \leq 0$. Here, we assume that $\widehat{\operatorname{deg}}\left(x^{2} \cdot \widehat{c}_{1}(H, k)^{d-1}\right)=0$. Then, $t=0$. Thus, $\left(z(x)_{K} \cdot H_{K}^{d-1}\right)=0$. So, by Theorem B, there is a positive integer $n$ and $u \in \widehat{\mathrm{CH}}^{1}\left(\operatorname{Spec}\left(O_{K}\right)\right)$ such that $n x=f^{\prime *}(u)$. We know $n x \cdot \widehat{c}_{1}(H, k)^{d}=0$, which implies $u \cdot f_{*}^{\prime}\left(\widehat{c}_{1}(H, k)^{d}\right)=0$. Therefore, $u=0$ in $\widehat{\mathrm{CH}}^{1}\left(\operatorname{Spec}\left(O_{K}\right)\right)_{\mathbb{Q}}$ because $f_{*}^{\prime}\left(\widehat{c}_{1}(H, k)^{d}\right)=\left(H_{K}^{d}\right)\left[\operatorname{Spec}\left(O_{K}\right)\right]$. Thus, $x=0$ in $\widehat{\mathrm{CH}}^{1}(X)_{\mathbb{Q}}$. This is a contradiction. Hence, we get $\widehat{\operatorname{deg}}\left(x^{2} \cdot \widehat{c}_{1}(H, k)^{d-1}\right)<0$. 


\section{Variants of Theorem B (non-abelian case)}

In this section, we will study variants of Theorem B or Theorem 1.1. The following theorem is a generalization of Theorem 1.1 to a higher rank vector bundle.

Theorem 3.1. Let $K$ be an algebraic number field and $O_{K}$ the ring of integers. Let $f: X \rightarrow \operatorname{Spec}\left(O_{K}\right)$ be an arithmetic variety and $(H, k)$ an arithmetically ample Hermitian line bundle on $X$. Assume that $d=$ $\operatorname{dim} f \geq 1$ and $X_{K}$ is smooth and geometrically irreducible. Let $(E, h)$ be a Hermitian vector bundle on $X$ such that $E_{\overline{\mathbb{Q}}}$ is semi-stable with respect to $H_{\overline{\mathbb{Q}}}$ and $\left(c_{1}\left(E_{K}\right) \cdot c_{1}\left(H_{K}\right)^{d-1}\right)=0$. Then, we have

$$
\widehat{\operatorname{deg}}\left(\widehat{\operatorname{ch}}_{2}(E, h) \cdot \widehat{c}_{1}(H, k)^{d-1}\right) \leq 0
$$

Moreover, if the equality holds, then $h_{\sigma}$ is Einstein-Hermitian with respect to a Kähler form $\Omega_{\sigma}=c_{1}\left(H_{\sigma}, k_{\sigma}\right)$ and $E_{\sigma}$ is flat for every $\sigma \in K(\mathbb{C})$.

Proof. Let $r$ be the rank of $E$. Since

$$
\widehat{c h}_{2}(E, h)=\frac{1}{2} \widehat{c}_{1}(E, h)^{2}-\widehat{c}_{2}(E, h)
$$

we have

$$
\begin{aligned}
\widehat{c h}_{2}(E, h) \cdot \widehat{c}_{1}(H, k)^{d-1}= & \frac{1}{2 r} \widehat{c}_{1}(E, h)^{2} \cdot \widehat{c}_{1}(H, k)^{d-1} \\
& -\left\{\widehat{c}_{2}(E, h)-\frac{r-1}{2 r} \widehat{c}_{1}(E, h)^{2}\right\} \cdot \widehat{c}_{1}(H, k)^{d-1} .
\end{aligned}
$$

By Lemma 8.2 of [Mo1], $E_{\sigma}$ is semistable with respect to $H_{\sigma}$. Thus the main theorem in [Mo2] implies that

$$
\widehat{\operatorname{deg}}\left(\left\{\widehat{c}_{2}(E, h)-\frac{r-1}{2 r} \widehat{c}_{1}(E, h)^{2}\right\} \cdot \widehat{c}_{1}(H, k)^{d-1}\right) \geq 0 \text {. }
$$

On the other hand, by Theorem 1.1, $\widehat{\operatorname{deg}}\left(\widehat{c}_{1}(E, h)^{2} \cdot \widehat{c}_{1}(H, k)^{d-1}\right) \leq 0$. Therefore, we have $\widehat{\operatorname{deg}}\left(\widehat{\operatorname{ch}}_{2}(E, h) \cdot \widehat{c}_{1}(H, k)^{d-1}\right) \leq 0$.

Next we consider equality condition.

We assume that $\widehat{\operatorname{deg}}\left(\widehat{\operatorname{ch}}_{2}(E, h) \cdot \widehat{c}_{1}(H, k)^{d-1}\right)=0$. First of all, by equality condition of the main theorem of $[\mathrm{Mo} 2], E_{\sigma}$ is flat for every $\sigma \in K(\mathbb{C})$. 
Let $h^{\prime}$ be an Einstein-Hermitian metric of $E$. Then, by Lemma 6.1 of [Mo1],

$$
\begin{gathered}
\widehat{\operatorname{deg}}\left(\left(\widehat{\operatorname{ch}}_{2}(E, h)-\widehat{\operatorname{ch}}_{2}\left(E, h^{\prime}\right)\right) \cdot \widehat{c}_{1}(H, k)^{d-1}\right)= \\
-\frac{(d-1) !}{4 \pi} \sum_{\sigma \in K(\mathbb{C})} D L\left(E_{\sigma}, h_{\sigma}, h_{\sigma}^{\prime}\right),
\end{gathered}
$$

where $D L$ is the Donaldson's Lagrangian. Therefore, we have

$$
\sum_{\sigma \in K(\mathbb{C})} D L\left(E_{\sigma}, h_{\sigma}, h_{\sigma}^{\prime}\right) \leq 0
$$

On the other hand, since $h^{\prime}$ is Einstein-Hermitian, we get $D L\left(E_{\sigma}, h_{\sigma}, h_{\sigma}^{\prime}\right) \geq$ 0 for all $\sigma \in K(\mathbb{C})$. Hence $D L\left(E_{\sigma}, h_{\sigma}, h_{\sigma}^{\prime}\right)=0$ for all $\sigma \in K(\mathbb{C})$. Thus $h_{\sigma}$ is Einstein-Hermitian for all $\sigma \in K(\mathbb{C})$.

In the case where $\operatorname{rk} E=1$, Theorem 1.1 says that if $\widehat{\operatorname{deg}}\left(\widehat{\operatorname{ch}}_{2}(E, h) \cdot \widehat{c}_{1}(H, k)^{d-1}\right)=0$, then $E_{K}$ is a torsion element of $\operatorname{Pic}^{0}\left(X_{K}\right)$. So we might expect a stronger property of $(E, h)$ than flatness. Here we introduce one notation. Let $M$ be a complex manifold and $F$ a flat vector bundle of rank $r$ on $M$. Let $\rho_{F}: \pi_{1}(M) \rightarrow \mathrm{GL}_{r}(\mathbb{C})$ be the representation of the fundamental group of $M$ arising from the flat vector bundle $F . F$ is said to be of torsion type if the image of $\rho_{F}$ is finite.

Proposition 3.2. Let $K$ be an algebraic number field and $O_{K}$ the ring of integers. Let $f: X \rightarrow \operatorname{Spec}\left(O_{K}\right)$ be an arithmetic variety, $H$ an $f$ ample line bundle on $X$ and $k$ a Hermitian metric of $H$. Assume that $d=\operatorname{dim} f \geq 1$ and $X_{K}$ is smooth and geometrically irreducible. Let $(E, h)$ be a Hermitian vector bundle of rank $r$ on $X$ such that $\left(E_{\sigma}, h_{\sigma}\right)$ is flat for each $\sigma \in K(\mathbb{C})$ and $\widehat{\operatorname{deg}}\left(\widehat{\operatorname{ch}}_{2}(E, h) \cdot \widehat{c}_{1}(H, k)^{d-1}\right)=0$. Let $\rho_{E_{\mathbb{C}}}: \pi_{1}\left(X_{\mathbb{C}}\right) \rightarrow$ $\mathrm{GL}_{r}(\mathbb{C})$ be the representation of the fundamental group of $X_{\mathbb{C}}$ arising from the flat vector bundle $E_{\mathbb{C}}$. If the image of $\rho_{E_{\mathbb{C}}}$ is abelian, then $E_{\sigma}$ is of torsion type for all $\sigma \in K(\mathbb{C})$.

Proof. We prove it by induction on $\operatorname{dim} X$. First, we consider the case $d=1$. Since the representation $\rho_{E_{\mathbb{C}}}$ is abelian, we have the decomposition $\rho_{E_{\mathbb{C}}}=\rho_{1} \oplus \cdots \oplus \rho_{r}$ such that $\operatorname{dim} \rho_{i}=1$ for all $i$. Therefore, there are flat line bundles $L_{1}^{\prime}, \ldots, L_{r}^{\prime}$ on $X_{\mathbb{C}}$ such that $E_{\mathbb{C}}=L_{1}^{\prime} \oplus \cdots \oplus L_{r}^{\prime}$. Thus, by an easy descent, we can find line bundles $L_{1}, \ldots, L_{r}$ on $X_{\overline{\mathbb{Q}}}$ such that $E_{\overline{\mathbb{Q}}}=L_{1} \oplus \cdots \oplus L_{r}$ and $\operatorname{deg}\left(L_{i}\right)=0$ for all $i$. Thus, by Proposition 10.8 in [Mo1], we have our assertion. 
Next, we assume that $d \geq 2$. Replacing $H$ by a higher multiple $H^{m}$ of $H$, we may assume that there is a section $\phi \in H^{0}(X, H)$ with the following properties:

i) $\operatorname{div}(\phi)_{K}$ is smooth and geometrically irreducible.

ii) If $\operatorname{div}(\phi)=Y+a_{1} F_{1}+\cdots+a_{s} F_{s}$ is the irreducible decomposition such that $Y$ is horizontal and $F_{i}$ 's are vertical, then $F_{i}$ 's are smooth fibers.

Since $\left(E_{\sigma}, h_{\sigma}\right)$ is flat for each $\sigma \in K(\mathbb{C})$, we have $\left(\operatorname{ch}_{2}(E) \cdot F_{i} \cdot H^{d-2}\right)=0$ and $\operatorname{ch}_{2}\left(E_{\sigma}, h_{\sigma}\right)$ is zero as differential form for every $\sigma \in K(\mathbb{C})$. Thus we have

$$
\widehat{\operatorname{deg}}\left(\widehat{\operatorname{ch}}_{2}(E, h) \cdot \widehat{c}_{1}(H, k)^{d-1}\right)=\widehat{\operatorname{deg}}\left(\widehat{\operatorname{ch}}_{2}\left(\left.(E, h)\right|_{Y}\right) \cdot \widehat{c}_{1}\left(\left.(H, k)\right|_{Y}\right)^{d-2}\right) .
$$

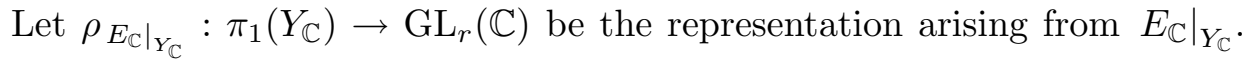
Since $\rho_{\left.E_{\mathbb{C}}\right|_{Y_{\mathbb{C}}}}$ is the composition of $\pi_{1}\left(Y_{\mathbb{C}}\right) \rightarrow \pi\left(X_{\mathbb{C}}\right)$ and $\rho_{E_{\mathbb{C}}}: \pi_{1}\left(X_{\mathbb{C}}\right) \rightarrow$

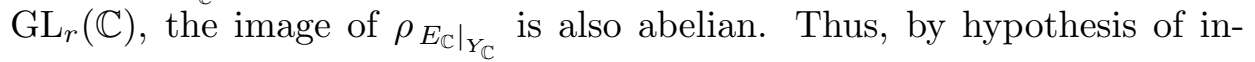
duction, $\left.E_{\sigma}\right|_{Y_{\sigma}}$ is of torsion type for every $\sigma \in K(\mathbb{C})$. On the other hand, by Lefschetz theorem, $\pi_{1}\left(Y_{\sigma}\right) \rightarrow \pi_{1}\left(X_{\sigma}\right)$ is surjective. Hence, $E_{\sigma}$ is also of torsion type for every $\sigma \in K(\mathbb{C})$.

Finally, we will pose two questions. Let $f: X \rightarrow \operatorname{Spec}\left(O_{K}\right)$ be a $(d+1)$ dimensional arithmetic variety, $(H, k)$ an arithmetically ample Hermitian line bundle on $X$, and $(E, h)$ a Hermitian vector bundle on $X$ such that $E_{\overline{\mathbb{Q}}}$ is semistable with respect to $H_{\overline{\mathbb{Q}}}$ and $\left(c_{1}\left(E_{K}\right) \cdot c_{1}\left(H_{K}\right)^{d-1}\right)=0$. An interesting problem is to find stronger equality conditions for

$$
\widehat{\operatorname{deg}}\left(\widehat{\operatorname{ch}}_{2}(E, h) \cdot \widehat{c}_{1}(H, k)^{d-1}\right) \leq 0 .
$$

Theorem 3.1 says that if $\widehat{\operatorname{deg}}\left(\widehat{\operatorname{ch}}_{2}(E, h) \cdot \widehat{c}_{1}(H, k)^{d-1}\right)=0$, then at least $E_{\sigma}$ is flat for every $\sigma \in K(\mathbb{C})$. Optimistically, one may pose the following question:

Question 3.3. If $\widehat{\operatorname{deg}}\left(\widehat{\operatorname{ch}}_{2}(E, h) \cdot \widehat{c}_{1}(H, k)^{d-1}\right)=0$, is $E_{\sigma}$ of torsion type for every $\sigma \in K(\mathbb{C})$ ?

By Proposition 3.2, if $\pi_{1}\left(X_{\mathbb{C}}\right)$ is abelian or $\operatorname{rk} E=1$, we have an affirmative answer of the above question. Moreover, if we carefully trace back the proof in Proposition 3.2, Question 3.3 can be reduced to the case $d=1$. So from now on, we assume that $d=1$. Let $\overline{\mathbf{M}}_{X_{K} / K}(r, 0)$ be the moduli scheme of semistable vector bundles on $X_{K}$ with rank $r$ and degree 0 . Let $h$ be a height function on $\overline{\mathbf{M}}_{X_{K} / K}(r, 0)$ arising from some ample line bundle on $\overline{\mathbf{M}}_{X_{K} / K}(r, 0)$. Our next question is 
Question 3.4. Are there constants $A$ and $B$ with the following properties?

(1) $A, B \in \mathbb{R}$ and $A>0$.

(2) For all semistable Hermitian vector bundle $(E, h)$ on $X$ with rank $r$ and degree 0 , we have

$$
h\left(E_{K}\right) \leq \frac{-A}{[K: \mathbb{Q}]} \widehat{\operatorname{deg}}\left(\widehat{c h}_{2}(E, h)\right)+B
$$

In some sense, Question 3.4 is related to Question 3.3. For, if $\widehat{\operatorname{deg}}\left(\widehat{c h}_{2}(E, h)\right)=0$ and Question 3.4 holds, then the height of $E_{K}$ is bounded. So $E_{K}$ should have some simple structure.

\section{References}

[BPV] W. Barth, C. Peters and A. Van de Ven, Compact complex surfaces, Ergebnisse der Mathematik und ihrer Grenzgebiete, 3.Folge · Band 4, Springer-Verlag, 1984.

[Fa] G. Faltings, Calculus on arithmetic surfaces, Ann. of Math. 119 (1984), 387-424.

[GS] H. Gillet and C. Soulé, Arithmetic analogs of the standard conjectures, Proc. Symp. in Pure Math. 55-I (1994), 129-140.

[Hr] P. Hriljac, Heights and Arakelov's intersection theory, Amer. J. Math. 107 (1985), 23-38.

[Ku] K. Künnemann, Some remarks on the arithmetic Hodge index conjecture, Comp. Math. 99 (1995), 109-128.

[Mi] J. Milnor, Morse Theory, Annals of Mathematic Studies, vol. 51, Princeton.

[Mo1] A. Moriwaki, Inequality of Bogolomov-Gieseker type on arithmetic surfaces, Duke Math. J. 74 (1994), 713-761.

[Mo2] _ Arithmetic Bogomolov-Gieseker's inequality, Amer. J. Math. 117 (1995), 1325-1347.

[Zh] S. Zhang, Positive line bundles on arithmetic varieties, Jour. A.M.S. 8 (1995), 187-221.

Department of Mathematics, Faculty of Science, Kyoto Univerity, KYOTO 606-01 JAPAN

E-mail address: moriwaki@kusm.kyoto-u.ac.jp 